\title{
Plough calculation peculiarities for walking tractors
}

\author{
Sergey Ovsyannikov ${ }^{1, *}$, and Evgeniy Kalinin² \\ ${ }^{1}$ Belgorod State Technological University named after V.G. Shukhov, 42, Kostyukova Street, Russia, \\ Belgorod, 308012 \\ ${ }^{2}$ Kharkiv National Technical University of Agriculture Petro Vasilenko, 44, Alchevski Street, \\ Ukraine, Kharkov, 62002
}

\begin{abstract}
The influence of forces and moments on a plowing walking tractor is considered. The main regularities in calculating the component forces of resistance to the tool in the mode of digging and changing the treatment depth are determined. It is established that physical efforts of the operator, aimed at regulating the depth of soil treatment, significantly exceed the permissible.
\end{abstract}

\section{Problem formulation}

Units based on motor blocks differ from traditional ones by rigid fixing of the tool to the frame of the motor block. Changing of the treatment depth in this case is carried out by pitching the whole unit. To do this, the operator moves the control bars in a vertical plane. Thus, the operator applies force to the control bars in the longitudinal and vertical directions, which leads to his rapid fatigue [1].

\section{Analysis of reference literature}

Calculation of the plough design parameters and the applied forces are considered in [2]. The interaction of the operator with the walking tractor, the action of forces and moments are discussed in detail in [3]. Based on the analysis of the reference literature, it is established that plowing walking tractors have distinctive features that need to be considered when performing calculations of design parameters.

\section{Object of work}

The object of work is to conduct an analytical foundation of the design parameters of plowing walking tractors and of their influence on the operator's physical participation in the traction movement.

\footnotetext{
* Corresponding author: ovsrg@mail.ru
} 


\section{Results of work}

The forces acting on the plough arise as a result of the soil resistance against the share's blade and its working surfaces during the operation of the plough-body. In each plane of projections, the total effect of elementary soil resistance forces on the body can be represented by a single resultant force (Figure 1) of a certain quantity and direction [3]. In the horizontal plane of projections (Fig. 1, a), the force $R_{x y}$ acts on the plough-body, forming the angle $\eta=\left(15-25^{\circ}\right)$ with the axis $x$.

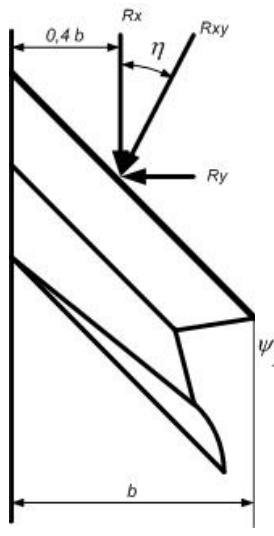

a

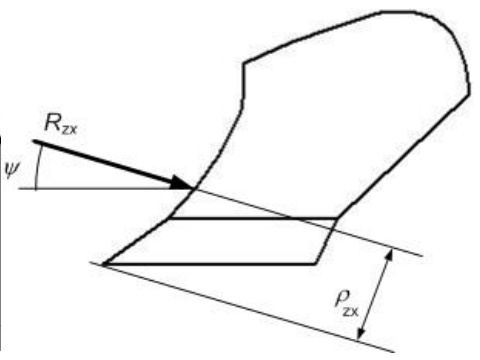

b

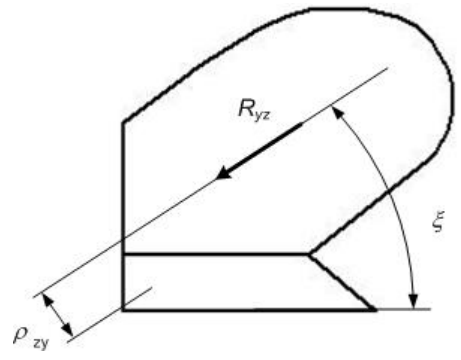

$\mathrm{c}$

Fig. 1. Diagram of the acting forces on the plough-body: $a-$ longitudinal-horizontal plane; $b-$ longitudinal-vertical plane; $c$ - transverse plane.

The force $R_{x y}$ crosses the share's blade at a distance equal to 0.4 of the plough width and 0.5 of the soil treatment depth. The components of the forces $R_{x y}$ on the $x$ and $y$ axes are determined by the correlations (1) and (2)

$$
\begin{aligned}
& R_{x}=\eta_{i \ddot{e}} \cdot k \cdot a \cdot b_{\hat{e}} \\
R_{Y}= & R_{X} \cdot \operatorname{tg} \eta=(0,25-0,45) R_{X} \approx \frac{R_{X}}{3}
\end{aligned}
$$

where $\eta_{\mathrm{pl}}$ is the plough efficiency, $\eta_{\mathrm{pl}}=0.7$;

$\mathrm{k}$ is the soil resistivity obtained by linear dynamometry of the plough;

$a, b_{\kappa}$ - the plough depth and width, respectively. According to agrotechnical requirements, the minimum depth of soil treatment should be $a=15 \mathrm{~cm}$, hence the plough width $b_{\kappa}=18$ $\mathrm{cm}$.

The soil resistivity $k$ depends on the soil type and structure, humidity and density. The average values of the soil resistivity after harvesting of the crops are: for heavy soils -90 $\mathrm{kPa}$, medium soils - $60 \mathrm{kPa}$, light soils - $30 \mathrm{kPa}$.

In the vertical projection plane, the force $R_{z x}$, forming an angle $\psi$ with the $x$-axis, acts on the plough-body (Fig. 1, б). On dense soils, the angle $\psi$ can have a negative value when a plough operates with a dull plough-share [3]. Considering modern technologies of self-sharpening of plough-shares, we assume that in the calculations the angle $\psi$ will be equal to $+12^{\circ}$ and on the overgrown sections $+8^{\circ}$. The vertical component of the force $R_{z x}$ is determined from the dependence (3). In the transverse plane, the projection of the force $R_{z x}$, which is equal to the vector sum of the forces $R_{z}$ and $R_{y}$ (4), acts on the body (Fig. 1, c). The direction of the force $R_{z y}$ is characterized by the value of the angle $\xi$, the tangent of which is determined by the ratio (5). The distance $\rho_{z y}$ from the nose of the share to the 
straight line, which is the continuation of the force $R_{z y}$, is approximately 0.5 of the arable layer depth for positive and 0.75 of the depth for negative values of the angle $\xi$.

$$
\begin{gathered}
R_{Z}=R_{X} \cdot \operatorname{tg} \psi \approx 0,2 \cdot R_{X} \\
R_{\mathrm{ZY}}=R_{X} \sqrt{\operatorname{tg} \eta^{2}+\operatorname{tg} \psi^{2}} \\
\operatorname{tg} \xi=\frac{\operatorname{tg} \eta}{\operatorname{tg} \psi}
\end{gathered}
$$

The forces acting on the walking tractor (Figure 2): $G_{M}$ is the motoblock weight force; $G_{o p}$ is the tool weight; $P_{o n}$ is the effort operator applies on the control bars in the vertical plane; $R_{x z}$ is the reaction of the soil to the tool in the longitudinal plane; $R_{x}$ is the horizontal component of $R_{x z} ; R_{z}$ is the vertical component of the force $R_{x z} ; S$ is the resultant force on the working parts of the tool.

To ensure self-digging of the plough-body, it is necessary for the resultant force $S$ to pass above the rotation axis of the wheels. Herewith, the moment $S \cdot \rho_{z x}$ is formed. The working parts of the tool are deepened until the plank reaches the furrow bottom, which leads to an increase of the soil reaction in the vertical direction up to leveling with the vertical load, thereby limiting further digging.

To determine the vertical component of the tool's weight force acting on the working parts, we will compose the equation for determining the tool's gravity center. Depending on the weight and location of the tool, ballast weights, the coordinates of the gravity center will change.

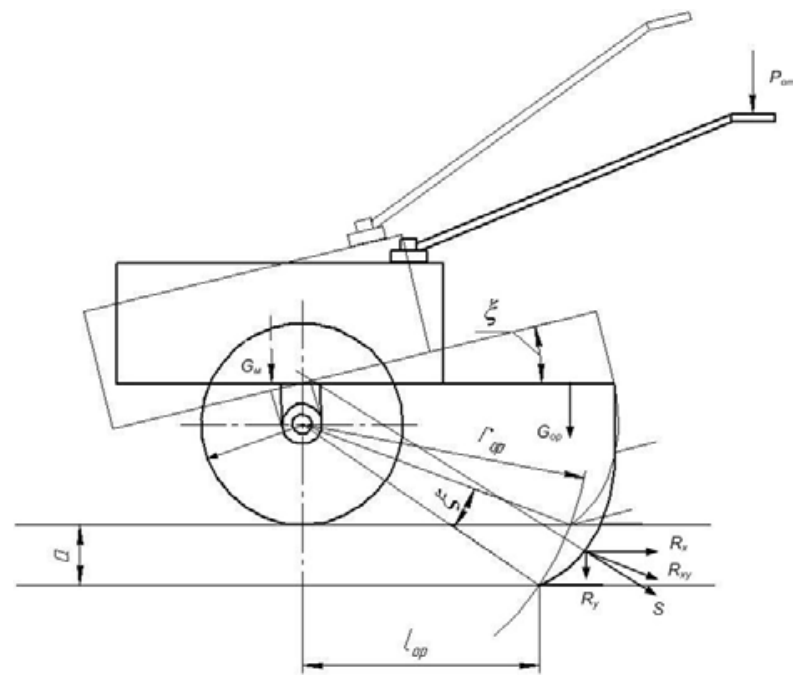

Fig. 2. The analytical model of a plowing walking tractor.

The longitudinal coordinate of the gravity center is determined from the equation (6). In the static position, the weight of the unit is balanced by the force of the operator $P_{o n}$ in the vertical direction on the control bars (7).

$$
x_{a}=\frac{\sum_{i=1}^{n} G_{i} \cdot x_{i}}{\sum_{i=1}^{n} G_{i}}
$$




$$
\mathrm{P}_{\text {ОП }}=\frac{G_{A} \cdot x_{A}}{x_{\text {ОП }}}
$$

where $G_{i}$ is the weight of the $i$ tool, device rigidly connected to the motor block;

$x_{i}$ is a longitudinal coordinate of the weight center of $i$ tool, device relative to the rotation axis of the wheels;

$x_{o n}$ is the longitudinal distance from the rotation axis of the wheels to the arms of the control bars.

During the movement the rotating torque develops on the wheels' axes of the walking tractor and it is equal to the moment resistance of the external forces. Accordingly, the reactive torque will act on the motor block frame and it will be numerically equal and opposite to the rotating torque. The action of this torque will increase the digging depth.

The rotating torque on the wheel axis will increase as the plough digs into the soil. When the nominal depth is reached, the plough resistance torque is not a constant value, as specific soil resistivity varies depending on soil structure and it is random. Consequently, the operator's efforts in the vertical plane on the motoblock's bars vary in proportion to the changes of rotating torque on the wheel drive axis.

To determine the values of the reactive torque, we consider the process of plough-body digging (Fig. 3). The soil reaction in the longitudinal plane will increase as the plough digs into the soil (8). The value of the vertical load is determined from the moment equation (9). The resultant force at the initial moment of digging is determined by the dependence (10). Herewith, a digging moment is formed (11) if it is directed clockwise relative to the wheel rotation axis, otherwise it is a shallowing moment. While digging, the direction of the soil reaction vector $R_{z x}$ deviates from the horizontal direction by an angle $\psi$, at the nominal position the angle is $\psi=12^{\circ}$ [3]. Then the resultant force $S$ is determined from the relation (12).

$$
\begin{gathered}
\mathrm{R}_{\mathrm{ZX}}=\eta_{n l} \cdot z \cdot b \cdot k \\
P_{Z} \cdot r_{\hat{i} \delta}=G_{a} \cdot x_{a}-M_{r}-P_{i \ddot{ }} \cdot x_{i i t},
\end{gathered}
$$

therefore

$$
\begin{gathered}
P_{Z}=\frac{G_{a} \cdot x_{a}-M_{r}-P_{\tilde{i}} \cdot x_{\tilde{i}}}{r_{i \dddot{i}}} . \\
S=\sqrt{R_{z x}^{2}+P_{z}^{2}} \cdot \\
M_{S}=S \cdot \rho_{Z X} \cdot \\
S=\sqrt{R_{Z X}^{2}+P_{Z}^{2}-2 R_{Z X}^{2} \cdot P_{Z} \cdot \cos (90-\psi)} .
\end{gathered}
$$

where $z$ is the plough depth, $0<z<a$. 


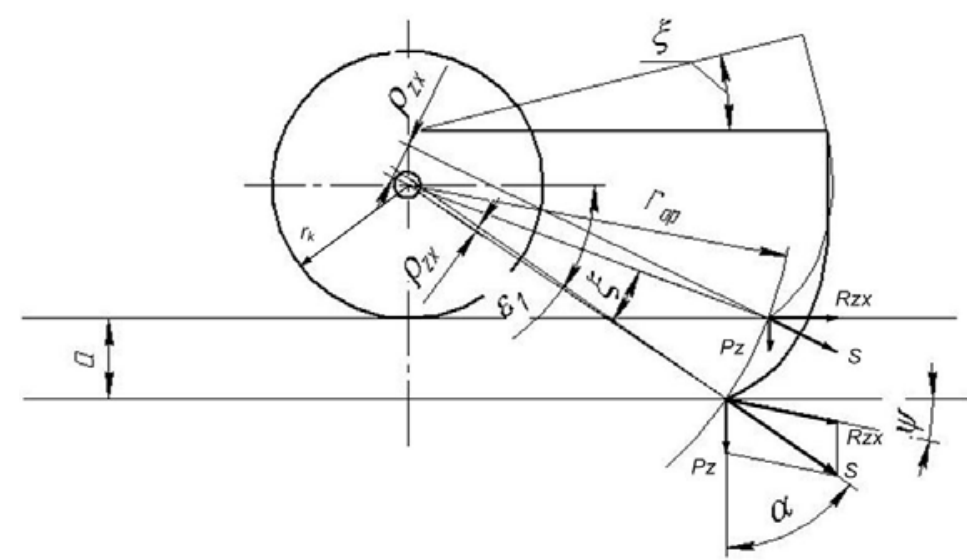

Fig. 3. The scheme of the forces acting on the working parts of the plough during digging.

In the process of digging, the angle $\alpha$ of the vector tilt of the resultant force $S$ changes. We determine the angle value using the formula (13), applying the sine theorem. The direction of the digging moment rotation is determined from the condition (14). The arm of the digging moment is determined by the formula (16).

$$
\frac{P_{Z}}{\sin \alpha}=\frac{S}{\sin \left(90^{\circ} \pm \psi\right)}, \text { where } 0<\psi<12^{\circ} .
$$

therefore

$$
\begin{aligned}
& \alpha=\arcsin \frac{P_{Z} \cdot \sin \left(90^{\circ}-\psi\right)}{S} \\
& \alpha<90^{\circ}-\xi_{1} \rightarrow^{+} \\
& \alpha>90^{\circ}-\xi_{1} \rightarrow^{-}
\end{aligned}
$$

where $\xi_{1}$ is the angle of tilting of the motor block in proportion to the plough digging:

$$
\begin{aligned}
& \xi_{1}=\arcsin \frac{r_{\hat{e}}+z}{r_{i \delta}} . \\
& \rho_{Z X}=r_{i \grave{i}} \cdot \sin \left(90-\xi_{1}-\alpha\right)
\end{aligned}
$$

As an example, the plowing walking tractor calculations were conducted on basis of Motor-Sich motoblock. As a result of calculations it was established that on medium and heavy soils with a treatment depth of more than $10 \mathrm{~cm}$, the operator is forced to limit the digging depth of the plough, moreover, the efforts on the control bars are much higher than it is permissible (more than $200 \mathrm{~N}$ ). In order to reduce the operator's force load, it is necessary to install support wheels on the plough.

\section{Conclusions}

The received mathematical model allows to define the basic parameters of a plough and a plowing walking tractor in general. As a result of calculations based on the obtained mathematical model, it is established that when working on plowing walking tractors, the operator has to apply considerable physical efforts to regulate the depth of plowing, and these efforts are twice or three times as high as the permissible ones. 
To reduce the physical efforts of the operator, it is necessary to equip the plowing walking tractors with support wheels.

The article was prepared within the framework of the Base University development based at V.G. Shukhov BSTU.

\section{References}

1. S. Ovsyannikov, Motrol 7, pp. 45-50 (2013).

2. Reference book for the designer of agricultural machines. In two volumes. Volume 2 / Edited by A.V. Krasnichenko, State scientific technical publication. Machine-building literature. M.: 1961. P. 5-19.

3. S. Ovsyannikov, Herald of National Technical University "Kharkiv Polytechnic Institute" 60. P. 25-30. (2012). 